\title{
Electrochemical Borylation of Carboxylic Acids
}

\author{
Lisa M. Barton ${ }^{\dagger}$, Longrui Chen ${ }^{\dagger}$, Donna Blackmond ${ }^{* \dagger}$, and Phil S. Baran ${ }^{* \dagger}$ \\ ${ }^{\dagger}$ Department of Chemistry, Scripps Research, 10550 North Torrey Pines Road, La Jolla, California 92037 \\ Supporting Information Placeholder
}

\begin{abstract}
A simple electrochemically mediated method for the conversion of alkyl carboxylic acids to their borylated congeners is presented. This protocol features an undivided cell setup with inexpensive carbonbased electrodes and exhibits a broad substrate scope and scalability in both flow and batch reactors. The use of this method in challenging contexts is exemplified with a modular formal synthesis of jawsamycin, a natural product harboring five cyclopropane rings.
\end{abstract}

Boronic acids are amongst the most malleable functional groups in organic chemistry as they can be converted into almost any other functionality. ${ }^{1}$ Aside from these versatile interconversions, their use in the pharmaceutical industry is

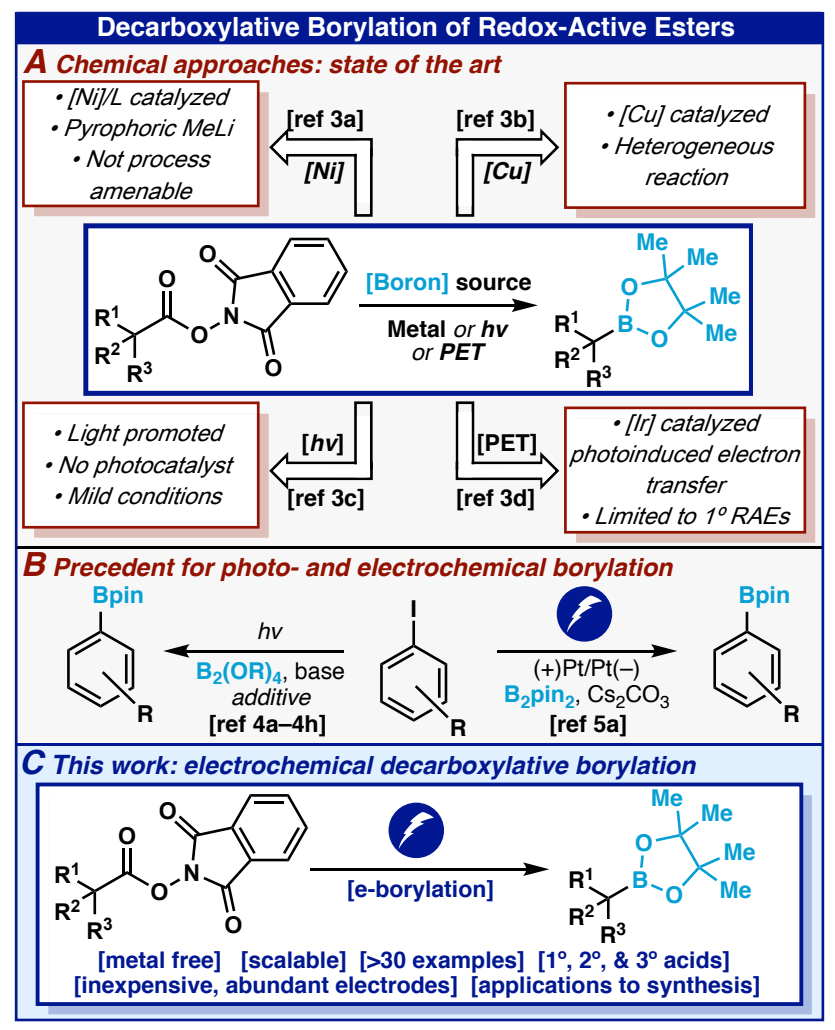

Figure 1. (A) Prior approaches to access alkyl boronic esters from activated acids. (B) Inspiration for initiating single electron transfer events electrochemically to achieve borylation. (C) Summary of this work.

gaining traction, resulting in approved drugs such as Velcade, Ninlaro, and Vabomere. ${ }^{2}$ It has been shown that boronic acids can be rapidly installed from simple alkyl carboxylic acids through the intermediacy of redox-active esters (RAEs) (Figure 1A). ${ }^{3}$ Our laboratory has shown that both $\mathrm{Ni}^{3 \mathrm{a}}$ and $\mathrm{Cu}^{3 \mathrm{~b}}$ can facilitate this reaction. Conversely, Li and Aggarwal both demonstrated photochemical variations of the same transformation. ${ }^{\mathrm{c}, \mathrm{d}}$ While these state-of-the-art approaches provide complementary access to alkyl boronic acids, each one poses certain challenges. For example, the requirement of excess boron source and pyrophoric MeLi under $\mathrm{Ni}$ catalysis is not ideal. Although more cost-effective and operationally simple, $\mathrm{Cu}$-catalyzed borylation conditions can be challenging on scale due to the heterogeneity resulting from the large excess of $\mathrm{LiOH} \cdot \mathrm{H}_{2} \mathrm{O}$ required. In addition to its limited scope, Li's protocol requires 4 equivalence of $\mathrm{B}_{2} \mathrm{pin}_{2}$ and an expensive Ir photocatalyst. The simplicity of Aggarwal's approach is appealing in this regard and represents an important precedent for the current study.

At the heart of each method described above, the underlying mechanism relies on a single electron transfer (SET) event to promote decarboxylation and form an alkyl radical species. In parallel, the related borylation of aryl halides via a highly reactive aryl radical can also be promoted by SET. While numerous methods have demonstrated that light can trigger this mechanism (Figure 1B), 4 simple electrochemical cathodic reduction can elicit the same outcome. 5 It was postulated that similar electrochemically-driven reactivity could be translated to alkyl RAEs. This disclosure reports a mild, scalable, and operationally simple electrochemical decarboxylative borylation (Figure $1 \mathrm{C}$ ) not reliant on transition metals or stoichiometric reductants. In addition to mechanistic studies of this interesting transformation, applications to a variety of alkyl RAEs, comparison to known decarboxylative borylation methods, and a formal synthesis of the polycyclopropane natural product jawsamycin ((-)-FR-900848) are presented.

Initial experiments with RAE $\mathbf{1}$ were promising, delivering ca. 20\% conversion to the borylated product. Extensive optimization of reaction conditions ultimately delivered $74 \%$ (64\% isolated) yield of product 2 (Figure 
2B). While the breadth of this experimentation can be found in the Supporting Information, outcomes from the screening of electron equivalents, solvent, electrolyte, current, electrode materials, and additives are showcased in Figure 2A. Experimentally, $1.8 \mathrm{~F} / \mathrm{mol}$ of RAE were needed to reach peak yields of $\mathbf{2}$; however, prolonged electrolysis resulted in erosion in yield due to observed product consumption (see SI).

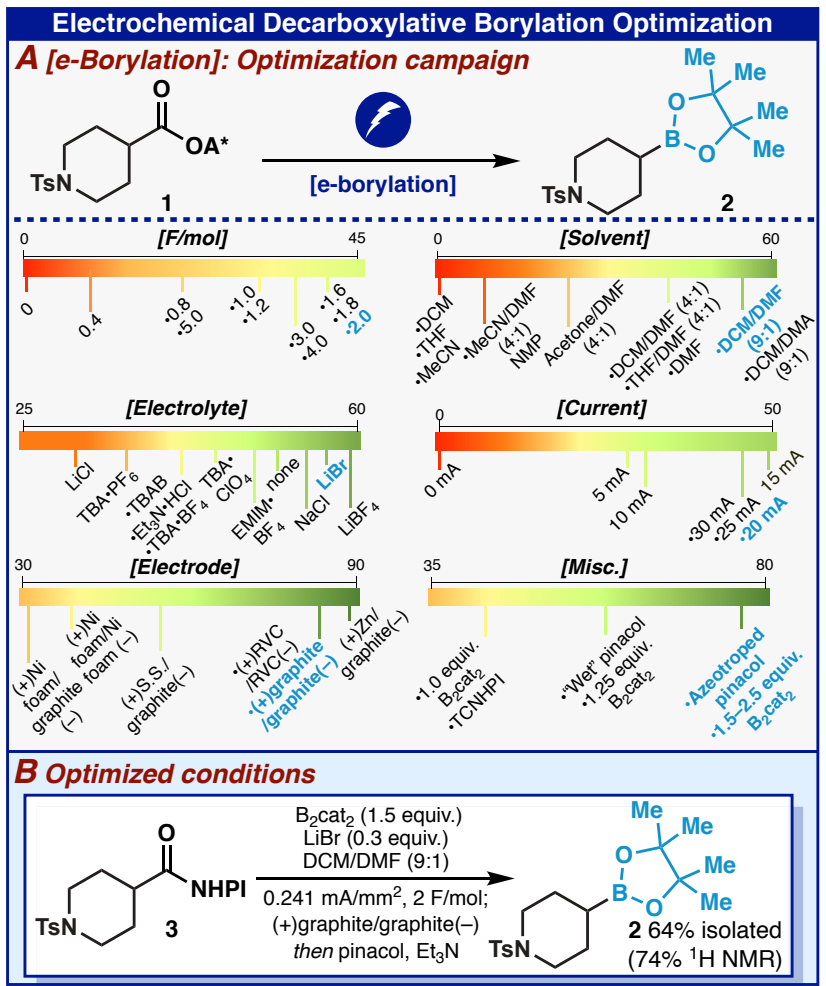

Figure 2. (A) Graphical overview of electrochemical decarboxylative borylation optimization. Modifications listed as a function of yield. (B) Optimized reaction conditions.

Solvent had the most profound influence on the reaction, as a balance of conductivity, Lewis-basicity, and polarity was needed to achieve high yields. In particular, the addition of DMF ( $\varepsilon=37)$ was vital, as it can act as a Lewisbase with $\mathrm{B}_{2} \mathrm{cat}_{2}{ }^{6}$ and undergo anodic oxidation ${ }^{7}$ making the overall reaction redox neutral, obviating the need for a sacrificial anode. A combination of two inexpensive graphite electrodes gave yields close to those obtained using a sacrificial $\mathrm{Zn}$ anode.

The choice of electrolyte also impacted the reaction, with $\mathrm{LiBr}$ and $\mathrm{LiBF}_{4}$ facilitating the best outcomes. Optimum results were observed when the reaction remained strictly anhydrous; therefore, $\mathrm{LiBr}$ was chosen as it is inexpensive and easily dried immediately prior to the reaction setup. On the $0.1 \mathrm{mmol}$ scale used for optimization, a current $>15$ $\mathrm{mA}$ was required for high yields, with the added benefit that higher currents significantly shorten the reaction time. An optimum current density of $0.241 \mathrm{~mA} / \mathrm{mm}^{2}$ was therefore identified, and holding this value constant was essential to achieving consistent yields when changing scale. The presence of any water during transesterification caused large inconsistencies in the yields, leading to a $\sim 20 \%$ loss in product. Therefore, pinacol was thoroughly azeotroped with toluene prior to use. Finally, a slight excess of $\mathrm{B}_{2} \mathrm{cat}_{2}$ (1.5 equivalents) was found to be necessary to achieve high yields.

With optimized conditions in hand, the scope of this transformation was explored on a wide range of acids (Figure 3A), often in yields equivalent to or higher than prior methods (comparative yields for $\mathrm{Ni}-, 3 \mathrm{a} \mathrm{Cu}-, 3^{\mathrm{b}} h v^{-, 3 \mathrm{c}, \mathrm{e}}$ and PET $^{3 \mathrm{~d}}$-based protocols are shown where reported). This method proved highly chemoselective towards borylation of RAEs, demonstrating a broad functional group tolerance including both aryl and alkyl halides (5$7, \mathbf{1 1}, \mathbf{1 6}, \mathbf{2 5})$, ketones $(\mathbf{3 1}, \mathbf{3 4})$, esters $(\mathbf{8}, \mathbf{1 0}, \mathbf{2 7 - 2 9})$, alkenes $(\mathbf{1 4}, \mathbf{3 0}, \mathbf{3 2})$, alkynes (12), protected amines (2, $\mathbf{1 5}, \mathbf{1 7}, \mathbf{1 8})$, and amino acids (10). RAEs derived from natural products and drug molecules smoothly underwent borylation, further highlighting the mildness of this method. Finally, bicyclic motifs commonly leveraged by medicinal chemists as phenyl bioisosteres ${ }^{8}$ were smoothly borylated using the developed conditions (27-29). While this protocol proved successful on a range of substrates, there remained some limitations. In particular, conformationally flexible tertiary, benzylic, and $\alpha$-heteroatom RAEs were nonproductive under these reaction conditions (see SI).

The protocol is highly scalable in an undivided cell, and on smaller scales can be run using a commercial potentiostat. These conditions were easily translated to larger scales in batch using Erlenmeyer flasks, homemade electrodes, and a large DC power supply (vide infra, Scheme 1C). Furthermore, the reaction was conducted on $100 \mathrm{~g}$ scale in a flow system, affording comparable yields to those obtained in batch (Figure $3 \mathrm{~B}$ ).

Following the exploration of this method's utility, preliminary studies were conducted to gain insight into the reaction mechanism. Cyclic voltammograms (CVs) were performed to ascertain the identity of the species undergoing cathodic reduction (Figure 4A). Interestingly, $\mathrm{B}_{2} \mathrm{cat}_{2}$ and the RAE independently exhibit reduction potentials in DMF at $-2.98 \mathrm{~V}$ and $-1.57 \mathrm{~V}$ respectively (relative to $\mathrm{Ag} / \mathrm{AgCl}$ ). However, when a 3:2 ratio of $\mathrm{B}_{2} \mathrm{cat}_{2}$ and RAE are premixed in DMF, a single reduction is observed at $-1.96 \mathrm{~V}$. This shift provides evidence for the existence of complex 37, initially proposed to be the active species under similar photochemical conditions. ${ }^{3 \mathrm{C}}$

Temporal measurements of reaction progress (Figure 4B) reveal that the reaction exhibits zero-order kinetics in $\left[\mathrm{B}_{2} \mathrm{cat}_{2}\right]$ and in [RAE] at higher concentrations. ${ }^{9}$ The slower rate at lower [RAE] may to be due to its consumption in unproductive reactions $(\mathrm{N}-\mathrm{O}$ cleavage leading to acid and decarboxylation/protodeborylation). Zero-order kinetics in both substrates suggests that the RAE rapidly complexes with $\mathrm{B}_{2} \mathrm{cat}_{2}$ in solution to form species 37 prior to electron transfer, with the subsequent electron transfer at the cathode being rate-determining (first-order). ${ }^{10}$ The observation of positive rate dependence on current, a feature that we have observed 
in previous electrochemical reactions, ${ }^{11}$ supports the proposal that the transfer of electrons at the cathode is rate limiting.

The presence of an intermediate alkyl radical was inferred by subjecting RAE $\mathbf{3 8}$ to the electrochemical borylation conditions (Figure $4 \mathrm{C}$ ). This resulted in an 8:1 ratio of linear (39) to cyclic (40) borylated products, arising from 5 -exo trig cyclization of the ensuing radical (rate constant $=1.0 \times 10^{5} \mathrm{~S}^{-1}$ ). ${ }^{12}$ Additionally, when RAE 41 (Figure 4C) underwent electrochemical borylation, only the homoallylic boronic ester $\mathbf{1 4}$ was isolated (rate constant = $\left.1.3 \times 10^{8} \mathrm{~S}^{-1}\right) .{ }^{12}$

\section{Electrochemical Decarboxylative Borylation}

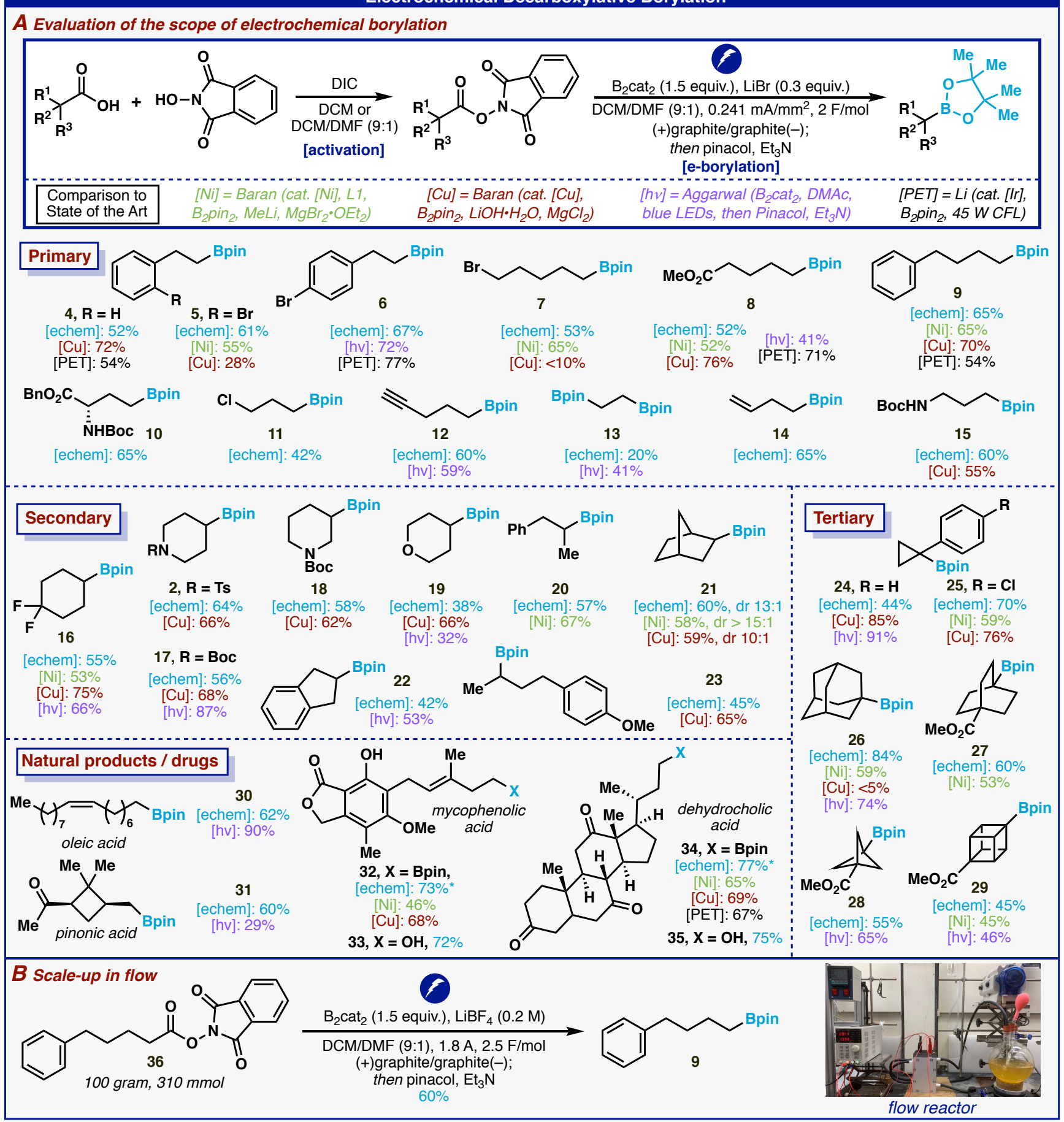

Figure 3. Scope and scale up of the electrochemical decarboxylative borylation. See Supporting Information for experimental procedures. Yields refer to isolated yields of products from corresponding RAE. *Refers to NMR yield of products that were directly oxidized to corresponding alcohol prior to isolation. 
Taken together, this evidence supports the mechanistic picture shown in Figure 4D. To begin, DMF complexes with one equivalent of $B_{2} c_{2} t_{2}$ to form 42, which can coordinate with an RAE to form the ternary complex $\mathbf{3 7}$. This species then undergoes single-electron reduction in the rate-limiting step at the cathode, yielding 43. Rapid fragmentation of 43 results in the liberation of 1 equivalent of $\mathrm{CO}_{2}, \mathbf{4 4}$, and alkyl radical 45. This radical can then react with another equivalent of 42, yielding the desired alkyl boronic ester 46. The liberated boroncentered radical $\mathbf{4 7}$ is most likely quenched, but in a minor pathway propagates a radical chain reaction by attacking a second RAE leading to $\mathbf{4 8}$, whose fragmentation would complete the cycle (see SI for further experimental investigation). This mechanistic picture closely mirrors Aggarwal's photochemical variant.3c

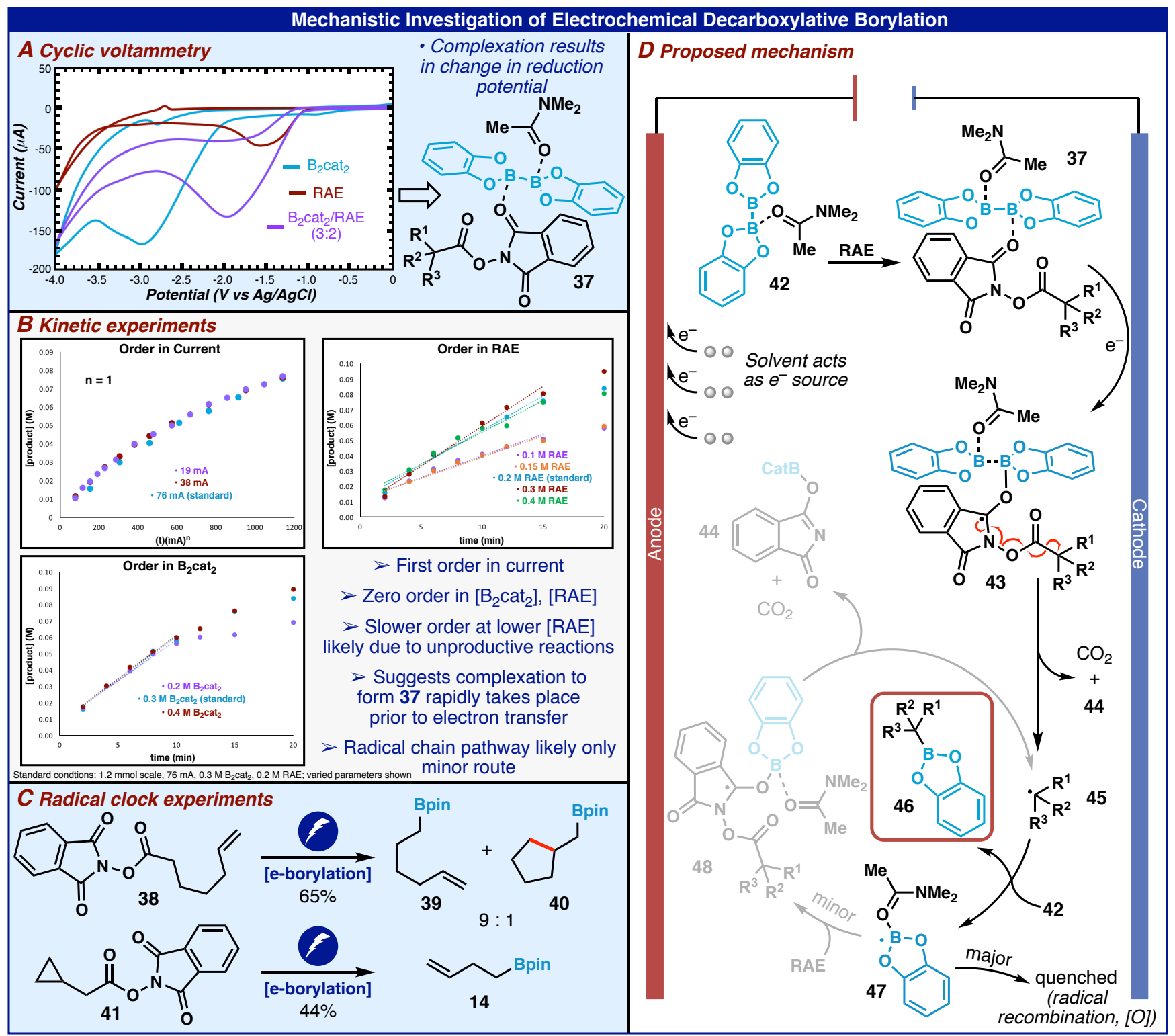

Figure 4. (A-C) Experimental investigation of mechanism. (D) Proposed mechanistic picture.

The kinetic results suggest a rationale for the enhanced efficiency of this electrochemical pathway with certain substrates. Generation of the free radical $\mathbf{4 5}$ from decarboxylation of $\mathbf{4 3}$ serves as entry into the cycle shown at the bottom of Figure 4D, which can theoretically propagate by continuous regeneration of $\mathbf{4 5}$. However, in this scenario, the reaction at the cathode serves only to initiate this stand-alone cycle. For this scenario to hold, an induction period should be observed, as the slow step occurs off-cycle at the cathode, and it becomes difficult to rationalize the positive order dependence on current. More likely is that the dominant route to product $\mathbf{4 6}$ lies in the continual generation of radical $\mathbf{4 5}$ via cathodic reduction and the radical-chain cycle represents only a minor route.

As a demonstration of the utility of this method, electrochemical borylation proved critical in accessing 
large quantities of key intermediates for a unique modular strategy en route to the polycyclopropane natural product jawsamycin ((-)-FR-900848, 57, Scheme 1A).13 All previous syntheses of this structurally fascinating natural product have commenced from olefins and relied on asymmetric cyclopropanations to build the polycyclopropane backbone. ${ }^{14}$ In contrast, this strategy represents a complimentary approach, hinging on the systematic coupling of chiral cyclopropane modules. This alternative retrosynthetic logic obviates the need for any asymmetric cyclopropanation step, as commercial building blocks such as $\mathbf{4 9}$ are easily desymmetrized to the corresponding hemiester, giving two differentiated synthetic handles which can be readily diversified through decarboxylative cross-coupling reactions. ${ }^{15}$

At the outset, borylation of the chiral RAE $\mathbf{5 0}$ proved incredibly challenging using prior methods (Scheme 1B).
Almost no product could be detected under Ni catalysis, and only modest yields were observed when employing either $\mathrm{Cu}$ or photochemical conditions. Even with the original optimized electrochemical protocol, only ca. $13 \%$ of the desired alkyl boronic ester was formed, with the mass balance corresponding to either hydrolyzed acid or decarboxylated byproduct. However, by utilizing electrodes with much higher surface areas (i.e. reticulated vitreous carbon, RVC) and activating the acid with the more electron deficient tetrachloro $\mathrm{N}$ hydroxyphthalimide derivative, workable yields of the desired product $\mathbf{5 1}$ were obtained (43\% by NMR, 35\% isolated). More importantly, this reaction was easily scaled (Scheme 1C), and up to 40 mmols of RAE could be processed in a single pass using a homemade batch reactor, with isolated yields up to $40 \%$ of the desired product $\mathbf{5 1 .}$

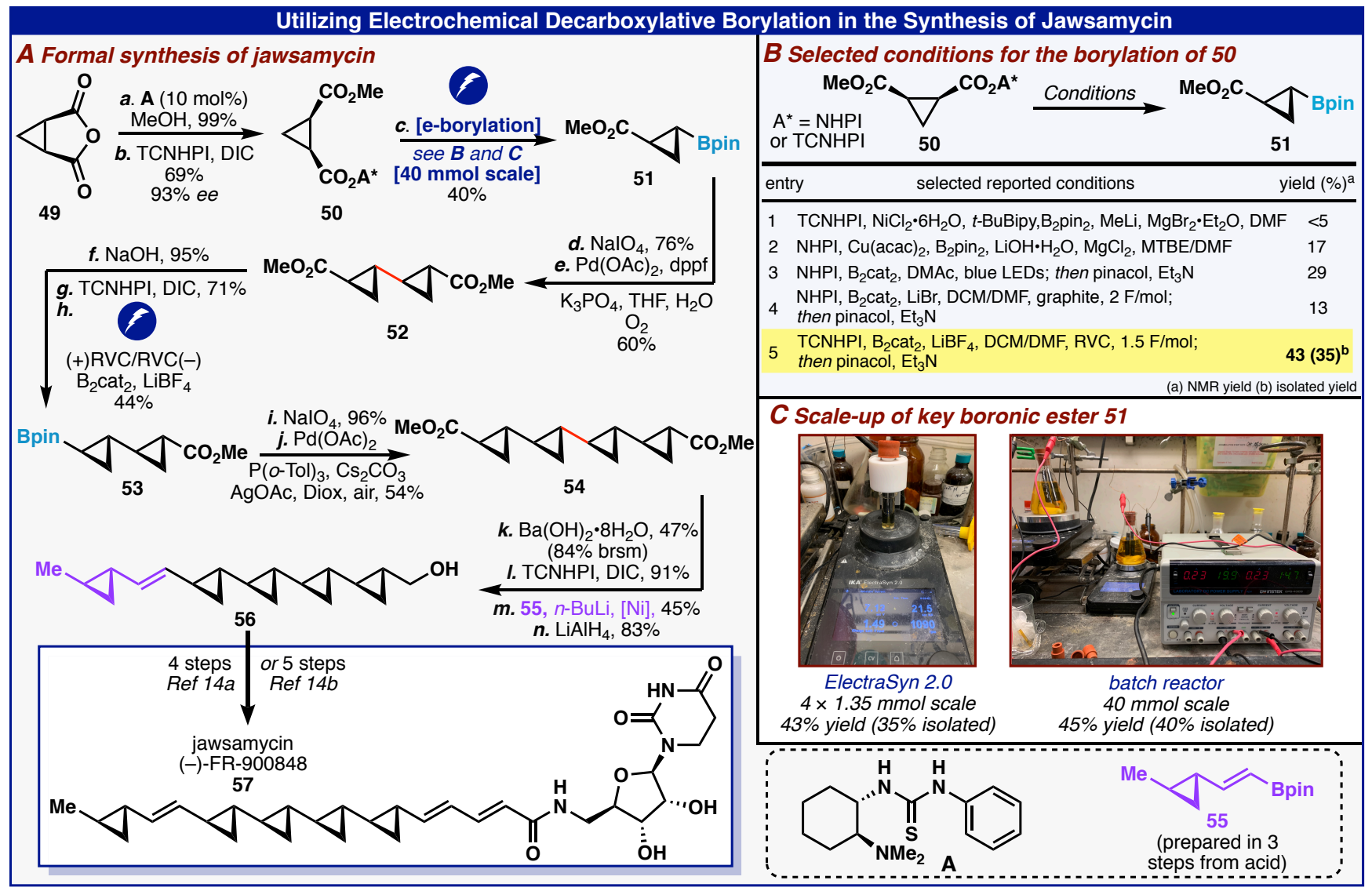

Scheme 1. (A) Formal synthesis of jawsamycin. (B) Examination of decarboxylative borylation conditions. (C) Scale up of borylation. See Supporting Information for reagents and conditions.

With sufficient quantities of $\mathbf{5 1}$ in hand, the boronic ester underwent oxidative cleavage to the corresponding acid, and the stage was set to explore Pd-catalyzed oxidative homocoupling. To the best of our knowledge, the homodimerization of cyclopropyl-boronic acids/esters has not been reported before. Although multiple boronic esters were screened, only the acid was sufficiently active under these conditions. Performing the homocoupling under an atmosphere of oxygen to re-oxidize Pd and complete the presumptive $\mathrm{Pd} / \mathrm{II} \mathrm{Pd}^{0}$ catalytic cycle ${ }^{16}$ proved critical to achieving reproducible results on scale (see SI for optimization summary).

Next, to form the tetra-cyclopropane $\mathbf{5 4}$, this sequence of borylation and homocoupling was repeated. Mono saponification of dimer 52, followed by activation, and a second electrochemical borylation, afforded boronic ester 53. After oxidative cleavage to the boronic acid however, we found that the original dimerization conditions resulted in no reaction; even on heating the reaction 
mixture to elevated temperatures, only protodeborylated product was observed. After extensive optimization (see $\mathrm{SI}$ ), rigorous exclusion of water using a $\mathrm{Cs}_{2} \mathrm{CO}_{3} /$ dioxane system, as well as utilizing the phosphine ligand $\mathrm{P}(o-\mathrm{Tol})_{3}$ began to result in product formation. The addition of AgOAc proved crucial here, ultimately corresponding to $54 \%$ of the desired tetramer 54. This dramatic improvement may arise from Ag serving dual roles by aiding the initial transmetalation of cyclopropyl boronic ester with $\mathrm{Pd}^{17}$ as well as functioning as an external oxidant. ${ }^{18}$

Mono saponification of the tetramer 54 and activation with TCNHPI set the stage for a final decarboxylative alkenylation with cyclopropyl vinyl-Bpin 55 (prepared in 3 steps, see SI). Using a slight modification to previously reported Ni-catalyzed Suzuki couplings of RAEs, ${ }^{15} 45 \%$ of

\section{ASSOCIATED CONTENT}

\section{Supporting Information}

The Supporting Information is available free of charge on the ACS Publications website.

Detailed experimental procedures and analytical data (PDF)

\section{AUTHOR INFORMATION}

\section{Corresponding Authors}

*pbaran@scripps.edu, *blackmond@scripps.edu

\section{ORCID}

Phil S. Baran: oooo-0001-9193-9053

\section{NOTES}

The authors declare no competing financial interest.

\section{ACKNOWLEDGMENT}

Financial support for this work was provided by the NIH (GM-118176). We are thankful to the NSF GRFP (predoctoral fellowship to L.M.B.), Bristol Myers Squibb (Graduate Fellowship to L.M.B.), and the ARCS Foundation (Graduate Scholarship to L.M.B.). We are grateful to Dr. Dee-Hua Huang and Dr. Laura Pasternack (Scripps Research) for assistance with NMR spectroscopy, Prof. A. L. Rheingold, Dr. M. Gembicky, and Dr. J. B. Bailey (UCSD) for X-ray crystallographic analysis, to Dr. Jason Chen, Brittany Sanchez, and Emily Sturgell (Scripps Automated Synthesis Facility) for assistance with HRMS, and LCMS, and to Yinghua $\mathrm{Xu}$ and Chengpu Chu for supplying flow equipment and engineering expertise. We thank Dr. David Hill (Scripps Research) for assistance with kinetics experiments and useful discussions, and Janice Dixon for assistance with manuscript preparation.

\section{REFERENCES}

1) (a) Boronic Acids: Preparation and Applications in Organic Synthesis, Medicine and Materials. 2nd ed.; Hall, D. G., Ed.; Wiley-VCH: Weinheim, Germany, 2011. (b) Sandford, C.; Aggarwal, V. K., Stereospecific Functionalizations and Transformations of Secondary and the desired vinylated product was obtained, which contained all five of the cyclopropane fragments of jawsamycin in place. A final reduction of the remaining ester completed the formal synthesis, intercepting the same strategic intermediate $\mathbf{5 6}$ used in both prior syntheses by Barrett and Falck.14a,b

To summarize, a practical electrochemical decarboxylative borylation of alkyl redox-active esters has been developed. The scope and mechanism of this transformation are similar to the photochemical variant and can be conveniently scaled up in batch or flow (100 g). Finally, this newly developed method proved essential for the preparation of a key cyclopropane intermediate en route to the polycyclopropanated natural product jawsamycin.

Tertiary Boronic Esters. Chem. Commun. 2017, 53, 54815494. (c) Zhu, C.; Falck, J. R., Transition-Metal-Free ipsoFunctionalization of Arylboronic Acids and Derivatives. Adv. Synth. Catal. 2014, 356, 2395-2410.

2) Plescia, J.; Moitessier, N., Design and Discovery of Boronic Acid Drugs. Eur. J. Med. Chem. 2020, 195, 112270.

3) (a) Li, C.; Wang, J.; Barton, L. M.; Yu, S.; Tian, M.; Peters, D. S.; Kumar, M.; Yu, A. W.; Johnson, K. A.; Chatterjee, A. K.; Yan, M.; Baran, P. S., Decarboxylative Borylation. Science 2017, 356, eaam7355. (b) Wang, J.; Shang, M.; Lundberg, H.; Feu, K. S.; Hecker, S. J.; Qin, T.; Blackmond, D. G.; Baran, P. S., Cu-Catalyzed Decarboxylative Borylation. ACS Catal. 2018, 8, 9537-9542. (c) Fawcett, A.; Pradeilles, J.; Wang, Y.; Mutsuga, T.; Myers, E. L.; Aggarwal, V. K., Photoinduced Decarboxylative Borylation of Carboxylic Acids. Science 2o17, 357, 283-286. (d) Hu, D.; Wang, L.; Li, P., Decarboxylative Borylation of Aliphatic Esters under Visible-Light Photoredox Conditions. Org. Lett. 2017, 19, 2770-2773. (e) For the translation of Aggarwal's conditions into flow and its application to the synthesis of 28: VanHeyst, M. D.; Qi, J.; Roecker, A. J.; Hughes, J. M. E.; Cheng, L.; Zhao, Z.; Yin, J., Continuous Flow-Enabled Synthesis of Bench-Stable Bicyclo[1.1.1]pentane Trifluoroborate Salts and Their Utilization in Metallaphotoredox Cross-Couplings. Org. Lett. 2020, 22, 1648-1654.

4) For examples of photochemical borylation of aryl halides: (a) Chen, K.; Wang, L.; Meng, G.; Li, P., Recent Advances in Transition-Metal-Free Aryl C-B Bond Formation. Synthesis 2017, 49, 4719-4730. (b) Mfuh, A. M.; Doyle, J. D.; Chhetri, B.; Arman, H. D.; Larionov, O. V., Scalable, Metal- and Additive-Free, Photoinduced Borylation of Haloarenes and Quaternary Arylammonium Salts. J. Am. Chem. Soc. 2016, 138, 2985-2988. (c) Chen, K.; Cheung, M. S.; Lin, Z. Y.; Li, P. F., Metal-free Borylation of Electron-rich Aryl (Pseudo)halides under Continuous-flow Photolytic Conditions. Org. Chem. Front. 2016, 3, 875-879. (d) Zhang, L.; Jiao, L., Visible-Light-Induced Organocatalytic Borylation of Aryl Chlorides. J. Am. Chem. Soc. 2019, 141, 9124-9128. (e) Mfuh, A. M.; Nguyen, V. T.; Chhetri, B.; Burch, J. E.; Doyle, J. D.; Nesterov, V. N.; Arman, H. D.; Larionov, O. V., Additive- and Metal-Free, Predictably 1,2and 1,3-Regioselective, Photoinduced Dual C-H/C-X Borylation of Haloarenes. J. Am. Chem. Soc. 2016, 138, 8408-8411. (f) Chen, K.; Zhang, S.; He, P.; Li, P., Efficient Metal-free Photochemical Borylation of Aryl Halides under Batch and Continuous-flow Conditions. Chem. Sci. 2016, 7 , 3676-3680. (g) Cheng, Y.; Mück-Lichtenfeld, C.; Studer, A., Metal-Free Radical Borylation of Alkyl and Aryl Iodides. 
Angew. Chem. Int. Ed Engl. 2018, 57, 16832-16836. (h) Jiang, M.; Yang, H.; Fu, H., Visible-Light Photoredox Borylation of Aryl Halides and Subsequent Aerobic Oxidative Hydroxylation. Org. Lett. 2016, 18, 5248-5251.

5) For examples of electrochemical borylation of aryl halides: (a) Hong, J. T.; Liu, Q. Y.; Li, F.; Bai, G. C.; Liu, G. Q.; Li, M.; Nayal, O. S.; Fu, X. F.; Mo, F. Y., Electrochemical Radical Borylation of Aryl Iodides. Chin. J. Chem. 2019, 37, 347-351. (b) Laza, C.; Duñach, E.; Serein-Spirau, F.; Moreau, J. J. E.; Vellutini, L., Novel Synthesis of Arylboronic Acids by Electroreduction of Aromatic Halides in the Presence of Trialkyl Borates. New J. Chem. 2002, 26, 373-375. (c) Laza, C.; Duñach, E., New Electrosynthesis of Arylboronic Esters from Aromatic Halides and Pinacolborane. Adv. Synth. Catal. 2oo3, 345, 580-583. (d) Laza, C.; Pintaric, C.; Olivero, S.; Duñach, E., Electrochemical Reduction of Polyhalogenated Aryl Derivatives in the Presence of Pinacolborane: Electrosynthesis of Functionalised Arylboronic Esters. Electrochim. Acta 2005, 50, 4897-4901.

6) Hioe, J.; Karton, A.; Martin, J. M.L; Zipse, H., Borane-lewis Base Complexes as Homolytic Hydrogen Atom Donors. Chemistry 2010, 16, 6861-6865.

7) Roth, H. G.; Romero, N. A.; Nicewicz, D. A., Experimental and Calculated Electrochemical Potentials of Common Organic Molecules for Applications to Single-Electron Redox Chemistry. Synlett 2016, 27, 714-723.

8) Mykhailiuk, P. K., Saturated Bioisosteres of Benzene: Where to Go Next? Org. Biomol. Chem. 2019, 17, 28392849.

9) The kinetics of the reaction were analyzed using reaction progress kinetic analysis (RPKA): (a) Blackmond, D. G., Reaction Progress Kinetic Analysis: A Powerful Methodology for Mechanistic Studies of Complex Catalytic Reactions. Angew. Chem. Int. Ed. 2005, 44, 4302-4320. (b) Mathew, J. S.; Klussmann, M.; Iwamura, H.; Valera, F.; Futran, A.; Emanuelsson, E. A. C.; Blackmond, D. G., Investigations of Pd-catalyzed ArX Coupling Reactions Informed by Reaction Progress Kinetic Analysis. J. Org. Chem.2006, 71, 4711-4722. (c) Blackmond, D. G., Kinetic Profiling of Catalytic Organic Reactions as a Mechanistic Tool. J. Am. Chem. Soc. 2015, 137, 10852-10866.

10) The precise kinetic order was determined by variable time normalization analysis: (a) Burés, J. A., Simple Graphical Method to Determine the Order in Catalyst. Angew. Chem. Int. Ed. 2016, 55, 2028-2031. (b) Nielsen, C. D. -T.; Burés, J., Visual Kinetic Analysis. Chem. Sci. 2019, 10, 348-353.

11) (a) Hu, P.; Peters, B. K.; Malapit, C. A.; Vantourout, J. C.; Wang, P.; Li, J.; Mele, L.; Echeverria, P. G.; Minteer, S. D.; Baran, P. S., Electroreductive Olefin-Ketone Coupling. $J$. Am. Chem. Soc. 2020, 142, 20979-20986. (b) Xiang, J.; Shang, M.; Kawamata, Y.; Lundberg, H.; Reisberg, S. H.; Chen, M.; Mykhailiuk, P.; Beutner, G.; Collins, M. R.; Davies, A.; Del Bel, M.; Gallego, G. M.; Spangler, J. E.; Starr, J.; Yang, S.; Blackmond, D. G.; Baran, P. S., Hindered Dialkyl Ether Synthesis with Electrogenerated Carbocations. Nature 2019, 573, 398-402. (c) Peters, B. K.; Rodriguez, K. X.; Reisberg, S. H.; Beil, S. B.; Hickey, D. P.; Kawamata, Y.; Collins, M.; Starr, J.; Chen, L.; Udyavara, S.; Klunder, K.; Gorey, T. J.; Anderson, S. L.; Neurock, M.; Minteer, S. D.; Baran, P. S., Scalable and Safe Synthetic Organic Electroreduction Inspired by Li-ion Battery Chemistry. Science 2019, 363, 838-845.
12) Griller, D.; Ingold, K. U., Free-Radical Clocks. Acc. Chem. Res. 1980, $13,317-323$.

13) (a) Yoshida, M.; Ezaki, M.; Hashimoto, M.; Yamashita, M.; Shigematsu, N.; Okuhara, M.; Kohsaka, M.; Horikoshi, K., A Novel Antifungal Antibiotic, FR-900848. I. Production, Isolation, Physico-chemical and Biological Properties. $J$. Antibiot. 1990, 43, 748-754. (b) Pietruszka, J., Synthesis and Properties of Oligocyclopropyl-containing Natural Products and Model Compounds. Chem. Rev. 2003, 103, 1051-1070.

14) For previous total syntheses: (a) Barrett, A. G. M.; Kasdorf, K., Total Synthesis of the Pentacyclopropane Antifungal Agent FR-900848. J. Am. Chem. Soc. 1996, 118, 1103011037. (b) Falck, J. R.; Mekonnen, B.; Yu, J. R.; Lai, J. Y., Synthesis of the Polycyclopropane Antibiotic FR-900848 via the Horeau Gambit. J. Am. Chem. Soc. 1996, 118, 60966097. For previous formal synthesis: (c) Verbicky, C. A.; Zercher, C. K., Olefin Cross-metathesis in the Preparation of Polycyclopropanes: Formal Synthesis of FR-900848. Tetrahedron Lett. 2000, 41, 8723-8727.

15) Chen, T. G.; Barton, L. M.; Lin, Y.; Tsien, J.; Kossler, D.; Bastida, I.; Asai, S.; Bi, C.; Chen, J. S.; Shan, M.; Fang, H.; Fang, F. G.; Choi, H.-w.; Hawkins, L.; Qin, T.; Baran, P. S., Building $\mathrm{C}(\mathrm{sp}(3))$-rich Complexity by Combining Cycloaddition and $\mathrm{C}-\mathrm{C}$ Cross-coupling Reactions. Nature 2018, 56o, 350-354.

16) Adamo, C.; Amatore, C.; Ciofini, I.; Jutand, A.; Lakmini, H., Mechanism of the Palladium-catalyzed Homocoupling of Arylboronic Acids: Key Involvement of a Palladium Peroxo Complex. J. Am. Chem. Soc. 2006, 128, 6829-6836.

17) For examples of Ag-enhanced transmetalations in Suzuki couplings: (a) Weibel, J. M.; Blanc, A.; Pale, P., Agmediated Reactions: Coupling and Heterocyclization Reactions. Chem. Rev. 2oo8, 108, 3149-73. (b) Lennox, A. J. J.; Lloyd-Jones, G. C., Selection of Boron Reagents for Suzuki-Miyaura Coupling. Chem. Soc. Rev. 2014, 43, 412443. (c) Zou, G.; Reddy, Y. K.; Falck, J. R., Ag(I)-promoted Suzuki-Miyaura Cross-couplings of $n$-Alkylboronic Acids. Tetrahedron Lett 2001, 42, 7213-7215. (d) Chen, J.; Cammers-Goodwin, A., 2-(Fluorophenyl)pyridines by the Suzuki-Miyaura method: $\mathrm{Ag}_{2} \mathrm{O}$ Accelerates Coupling Over Undesired Ipso Substitution (SNAr) of Fluorine. Tetrahedron Lett 2003, 44, 1503-1506. (e) Korenaga, T.; Kosaki, T.; Fukumura, R.; Ema, T.; Sakai, T., SuzukiMiyaura Coupling Reaction using Pentafluorophenylboronic Acid. Org. Lett. 2oo5, 7, 49154917.

18) For examples of using $\mathrm{Ag}$ as a terminal oxidant in Pdcatalyzed oxidative couplings: (a) Funes-Ardoiz, I.; Maseras, F., Oxidative Coupling Mechanisms: Current State of Understanding ACS Catal. 2018, 8, 1161-1172. (b) Stuart, D. R.; Villemure, E.; Fagnou, K., Elements of Regiocontrol in Palladium-catalyzed Oxidative Arene Cross-coupling. $J$. Am. Chem. Soc. 2007, 129, 12072-12073. (c) Zhao, J.; Huang, L.; Cheng, K.; Zhang, Y., Palladium-catalyzed Alkenation of Thiophenes and Furans by Regioselective CH Bond Functionalization. 2oo9, 5o, 2758-2761. (d) Wen, P.; Li, Y.; Zhou, K.; Ma, C.; Lan, X.; Ma, C.; Huang, G., Palladium-catalyzed $\mathrm{C}-2$ selective $\mathrm{C}-\mathrm{H}$ Olefination of Pyridines. Adv. Synth. Catal. 2o12, 354, 2135-2140. (e) Zhao, B., Pd-catalyzed C-3 Functionalization of Indolizines via $\mathrm{C}-\mathrm{H}$ Bond Cleavage. Org. Biomol. Chem. 2012, 10 , 7108-7119. 


\section{Table of Contents}

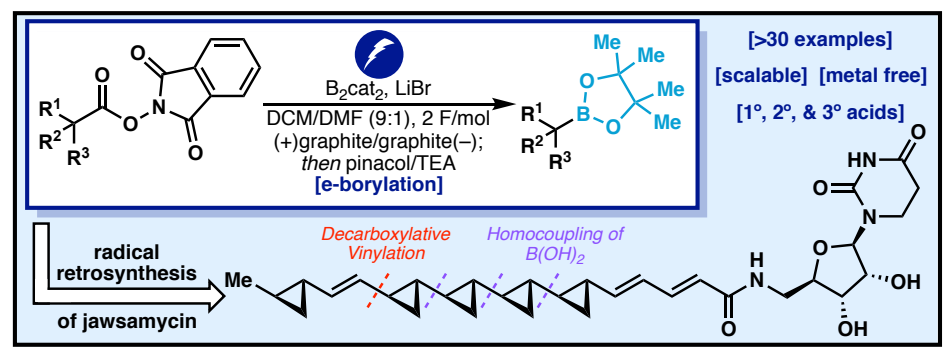

